\title{
The Moderator of Innovation Culture and the Mediator of Realized Absorptive Capacity in Enhancing Organizations' Absorptive Capacity for SPI Success
}

\author{
Jung-Chieh Lee, International Business Faculty, Beijing Normal University Zhuhai, Zhuhai, China \\ Chung-Yang Chen, National Central University, Jhong-Li, Taiwan
}

\begin{abstract}
Software process improvement (SPI) is critical to information system development. In the context of successful SPI, this research focuses on a firm's dynamic learning ability to see how it facilitates an effective means of acquiring and utilizing external SPI knowledge in responding to changing software development environments. Specifically, the authors propose a research model to investigate how two mechanisms of absorptive capacity are incorporated with innovation culture as a contextual factor to enable successful software process improvement. A survey was conducted including 125 SPI certified firms in China and Taiwan to examine the model. The findings indicate that a firm's potential absorptive capacity significantly influences realized absorptive capacity, which has a significant impact on SPI success and acts as a partial mediator between potential absorptive capacity and SPI success. Moreover, the results suggest that the mediating effect of potential absorptive capacity on SPI success via realized absorptive capacity is amplified when innovation culture is imposed.
\end{abstract}

\section{KEYWORDS}

Capability Maturity Model Integration, Dynamic Capability, Innovation Culture, Potential Absorptive Capacity, Realized Absorptive Capacity, Software Process Improvement (SPI)

\section{INTRODUCTION}

Software process improvement (SPI) is particularly important for firms and business units because it enhances and sustains their competitive advantage in the business market (Lee et al., 2016). Software development is knowledge intensive in innovation and mutual learning and often takes advantage of external sources to advance software development processes (Matusik and Heeley, 2005). Therefore, SPI implementation often relies on SPI knowledge, skills, expertise, experience, methodologies, technical support from external sources - e.g., external mediating institutions, such as SPI consulting firms and vendors - and external knowledge bodies, such as the Capability Maturity Model Integration (CMMI) and the International Standards Organization (ISO), to address challenges that arise during implementation (Feher and Gabor, 2006; Meehan and Richardson, 2002). 
Based on organizational learning theory (March, 1991), SPI is commonly recognized as an organizational learning process because the exploration of external process knowledge and the exploitation of existing process knowledge in organizations play critical roles in SPI implementation (Rus and Lindvall, 2002; Dyba, 2005; Lee et al., 2017). However, successful SPI relies on how effectively a firm can internalize these external lessons (Mathiassen and Pourkomeylian, 2003; Alagarsamy et al., 2008). Furthermore, software processes are executed in dynamic development and turbulent business environments (Xu and Ramesh, 2007). Thus, SPI requires continual endeavours in order for firms to maintain their competence. However, the existing literature does not fully grasp how to address a firm's learning ability to internalize external SPI knowledge nor addresses how organizational learning continually supports changing SPI needs under dynamic environments.

This study focuses on dynamic capabilities theory to address continual learning ability in SPI. Dynamic capabilities refer to a firm's abilities to adapt, renew, and reconfigure internal and external competences to address rapidly changing environments (Teece et al., 1997; Zahra et al., 2006). Zahra and George (2002) extended dynamic capability to include absorptive capacity (AC), which represents a firm's dynamic ability to acquire, assimilate, and apply knowledge from external environments. $\mathrm{AC}$ enables a firm to renew or reconfigure its existing knowledge stock, creating new knowledge, processes, or products to better match rapidly changing environments (Jansenet al., 2005; Volberda et al., 2010). It can be considered a specific organizational learning process for learning, implementing, and disseminating external knowledge internally in order to strengthen, complement, or refocus the firm's knowledge mechanisms (Zahra and George, 2002; Lane et al., 2006; Volberda et al., 2010; Sun and Anderson, 2010; Roberts et al., 2012). Peng et al. (2014) showed that firms with greater AC can quickly and precisely recognize and assimilate the value of external knowledge and information, which then allows them to adopt ad hoc information technology more quickly.

To gain a better understanding of a firm's ability to acquire and utilize external SPI knowledge, this study adopts the two categories of AC developed by Zahra and George (2002) - potential AC (PAC) and realized AC (RAC) - and investigates how these types of AC influence SPI success. PAC represents a firm's ability to identify, acquire, embrace, and assimilate external knowledge, while RAC refers to its ability to leverage newly acquired knowledge and incorporate transformed knowledge into the development of innovation processes and operations (Fosfuri and Tribo, 2008). In the existing information systems (IS) literature, Saraf et al. (2013) indicated that PAC helps a focal firm acquire and understand external knowledge that is specific to the enterprise's information systems, such as enterprise resource planning (ERP), while RAC provides a firm with the ability to exploit the absorbed knowledge to facilitate greater ERP usage. In the context of SPI, a firm's AC is expected to be the enabler that continually turns external SPI knowledge into internal development processes to reflect the environmental changes in software development. Accordingly, insight into a firm's PAC and RAC is essential to understand how SPI knowledge acquisition and utilization affects SPI success.

The knowledge-based view (KBV) suggests that contextual factors, i.e., corporate culture, impact how knowledge is acquired and employed in firms (Abubakre et al., 2017; Alavi and Leidner, 2006; Lee et al., 2011; Castro et al., 2013) and play an essential role in a firm's knowledge-intensive software development (Jones et al., 2006; Leidner and Kayworth, 2006; Ke and Wei, 2008; Moreno et al., 2012). Nonaka and Takeuchi (1995) considered innovation culture (IC) to be the most important contextual factor influencing a firm's knowledge mechanisms. IC has been shown to enhance the acquisition and application of new knowledge, to improve a firm's knowledge stock and to help achieve organizational objectives (Castro et al., 2013). However, in the context of SPI, little research investigates how IC might be associated with PAC or RAC.

Therefore, we address the following research questions:

1. How do the mechanisms of AC in terms of PAC and RAC help a firm achieve SPI success?

2. How does IC influence the contexts of PAC and RAC? 
We propose a research model that links PAC, RAC, IC, and SPI success by using a quantitative empirical survey of SPI certified firms in the Asia-Pacific region and emphasizing Taiwan and mainland China as the primary data source. Specifically, this study adopts partial least squares (PLS), a structural equation modelling approach, to test the proposed model empirically, analysing 125 samples of organizations with the specific SPI program Capability Maturity Model Integration (CMMI) (CMMI Institute, 2010). The rest of this study is organized as follows. Section 2 reviews relevant research studies on SPI, AC, and IC, and proposes corresponding research hypotheses. Section 3 provides details on the data collection, including population and sampling. Section 4 examines the proposed research model and explores relationships between the hypotheses. Section 5 summarizes the findings and discusses the outcomes. Finally, we describe the limitations of this study and outline directions for future research in Section 6.

\section{THEORETICAL BACKGROUND AND HYPOTHESES DEVELOPMENT}

\subsection{Software Process Improvement Success}

SPI helps firms integrate traditional organizational functions and sets process improvement goals and priorities that update existing process systems to improve organizational performance (Shih and Huang, 2010; Lee et al., 2016). SPI has played a critical role in helping firms achieve various business benefits, e.g., improving product quality, reducing time to market, improving productivity, and reducing costs. To realize these benefits, effective SPI implementation requires time, careful scheduling, resources, and knowledge (Meehan and Richardson, 2002; Mathiassen and Pourkomeylian, 2003; Niazi et al., 2006). Decisions about SPI implementation are influenced by organizational factors, and several studies have analysed the critical success factors for SPI (Rainer and Hall, 2002; Dyba, 2005; Niazi et al., 2006; Sulayman et al., 2014). Specifically, Dyba (2005) empirically investigated software organizations to examine the influence of organizational factors on successful SPI implementation and discussed six organizational factors, including the exploitation of existing knowledge and the exploration of new knowledge. Several recent studies have also proposed similar findings to explain the importance of SPI knowledge in an SPI context. For example, Larrucea et al. (2016) indicated that firms should gather useful knowledge and the experiences of others to help them deploy appropriate processes to best meet their needs. Uskarci and Demirors (2017) stated that employees must possess satisfactory knowledge regarding SPI activities to support them and participate in teams working to improve organizational processes.

Dyba (2005) validated a theoretical model of SPI success factors and proposed an operational definition of variables for this success. The study suggested that SPI success was defined by two indicators: improved organizational performance and the perceived level of SPI success, including cost reduction, decreased cycle time, and increased customer satisfaction. Dyba's theoretical model has been applied in various studies. Sulayman et al. (2014) used Dyba's work to develop a specialized framework of SPI success factors for small web companies. Winter and Ronkko (2010) investigated product usability metrics, adopting Dyba's (2005) SPI success factors. Based on Dyba's (2005) work, Egorova et al. (2009) evaluated the effect of software engineering practices for industrial projects. In this study, we adopt Dyba's (2005) definition of SPI success as the dependent variable in the proposed model.

\subsection{Absorptive Capacity}

AC has played a critical role in investigations of IT and IS (Roberts et al., 2012; Sharma et al., 2012; Saraf et al., 2013). While AC was originally defined as a firm's ability to recognize the value of, assimilate, and apply new external information to commercial ends (Cohen and Levinthal, 1990, p. 128), it also implies learning and acting in discovering scientific and technological activities outside the organization's limits (Zahra and George, 2002; Lee et al., 2018). Therefore, AC enables firms 
to achieve superior organizational performance, innovation capability, and competitive advantage (Jansen et al., 2005; Lane et al., 2006; Lee et al., 2017). Generally, AC is treated as a dynamic capability that affects a firm's ability to reconfigure its existing substantive capabilities (Zahra and George, 2002; Jansen et al., 2005). Thus, AC helps firms flexibly generate new valuable knowledge in turbulent environments by reshaping and renewing its existing knowledge stock (Pavlou \& El Sawy, 2006; Roberts et al., 2012). Zahra and George (2002) further distinguished four dimensions of AC: acquisition, assimilation, transformation, and exploitation. Each dimension is considered a capability that helps to produce the organizations' dynamic capabilities (Sun and Anderson, 2010), and these dimensions explain how AC influences a firm's knowledge mechanisms when the opportunity or need arises (Camisón and Forés, 2010; Lee et al., 2017).

Specifically, acquisition refers to a firm's ability to identify, acquire, and value external knowledge that is critical to operations, while assimilation refers to a firm's ability to analyse, process, interpret, and understand the acquired knowledge. According to several studies, knowledge acquisition and assimilation combine to form PAC, a type of AC that enables a firm to identify, filter and then acquire new valuable external knowledge for future use and application (Lee et al., 2017; Zahra \& George, 2002). Transformation is a firm's ability to combine existing knowledge and newly acquired and assimilated knowledge for future use, while exploitation refers to its ability to integrate acquired, assimilated, and transformed knowledge into its operations to develop new processes, routines, operations, and systems. After implementing PAC, a firm's RAC transforms and exploits the absorbed knowledge by utilizing and applying it to support innovation (Lee et al., 2017; Zahra \& George, 2002).

PAC and RAC represent two separate yet complementary roles of AC to facilitate the use of new external knowledge (Zahra and George, 2002). According to Saemundsson and Candi (2017), PAC is critical because it allows firms to acquire and assimilate externally generated knowledge and helps them recognize opportunities in a business environment. However, RAC is an essential foundation for firms to leverage the absorbed knowledge to develop new products, services or processes (Fosfuri \& Tribo, 2008; Leal-Rodríguez et al., 2014). As Leal-Rodríguez et al. (2014) stated, firms cannot take advantage of knowledge without first acquiring it. Conversely, firms may possess the capability to acquire and assimilate knowledge but may not transform and exploit this knowledge for profit generation.

The literature has shown the importance of AC in IT/IS deployment and implementation. For example, Harrington and Guimaraes (2005) indicated that AC establishes an external communication channel to gather useful knowledge that influences implementation of new technologies. Sharma et al. (2012) showed that AC acts an important facilitator to help ensure effectiveness and efficiency during the highly customized and learning-intensive process of ERP systems adoption. Bharati et al. (2014) noted that a higher level of AC contributes to a greater assimilation of social media technologies. Wei et al. (2015) indicated that AC can help organizations perceive the commercial potential of technological breakthroughs. Thus, strong AC is critical for organizations' successful technological adoption.

\subsection{Absorptive Capacity and SPI Success}

During SPI implementation, the acquisition capability of PAC first enables a firm to identify and gather relevant SPI information and knowledge, such as best practices, methodologies, and technical support, from external sources (i.e., SPI consulting firms and vendors; Lee and Chen, 2017). Knowledge acquisition helps the firm recognize, value and capture adequate and useful SPI knowledge to meet and satisfy the firm's SPI demands, depending on its specific characteristics, such as technology, systems, people, and processes (Lee et al., 2018). In the next stage of PAC, the assimilation function addresses the acquired SPI knowledge by converting the knowledge into a comprehensible and meaningful format for the company and then disseminating it across the firm to ensure effective SPI implementation. 
Nevertheless, acquiring knowledge alone is not enough for complete SPI implementation. The firm may need further RAC to exploit and embed this knowledge when implementing ad hoc process improvement situations (Albort-Morant et al., 2018; Joshi et al., 2010; Leal-Rodríguez et al., 2014). Regarding the contextual effect between PAC and RAC, Joshi et al. (2010) demonstrated that a firm's PAC can help strengthen its RAC because PAC gathers considerable amounts of external knowledge to be filtered, harvested and analysed into local formats to prepare RAC to take effect. In other words, for the absorption of external SPI knowledge, PAC helps a firm to acquire and assimilate suitable external knowledge that can be used and applied to a firm's operations via its RAC. Thus, we hypothesize the following:

H1: Potential absorptive capacity has a positive influence on realized absorptive capacity.

In the context of this study, RAC is reflected in the transformation and exploitation of SPI knowledge. Specifically, in the first stage of RAC, knowledge transformation aims to combine a firm's existing knowledge and its newly absorbed SPI knowledge. Transformation acts by integrating the newly acquired knowledge about SPI practices into the firm's software development operations. As a result of transformation, internal and existing SPI knowledge may be synthesized through the addition or elimination of knowledge or the conversion of external knowledge (Lee \& Chen, 2018, p. 24). Knowledge exploitation not only helps the firm leverage the transformed SPI knowledge into its software development routines but also renews and rearranges the software processes that are meant to increase the effectiveness of the development. Thus, RAC can provide support for SPI activities and upgrade the firm's software engineering competence by increasing the efficiency of software development and customers' satisfaction with the result. As such, RAC seems to be critical for SPI success. Thus, we hypothesize the following:

H2: Realized absorptive capacity has a positive influence on SPI success.

Zahra and George (2002) argued that external knowledge may not be transformed and exploited until it has been acquired and assimilated. Further, SPI is often aided by external knowledge (Ravichandran and Rai, 2003). As discussed above, AC development could enhance SPI knowledge acquisition and utilization, and the implementation of SPI first requires the effective acquisition of external knowledge (i.e., PAC). However, external knowledge cannot affect SPI success if the mechanism to transform and embed the absorbed knowledge into the firm's real processes, operations, and routines is not established (i.e., RAC). Thus, PAC is the first step to acquire external SPI knowledge and RAC is the next logical step to exploit this new SPI knowledge. This argument is logically consistent with Albort-Morant et al.'s (2018) study, which indicated that an organization's competence in acquiring and exploiting external valuable knowledge is essential in the new product development context. They revealed a full meditation relationship between PAC, RAC and new product development performance and further confirmed that even if PAC exists, RAC is necessary to further utilize the acquired external knowledge and achieve greater new product development performance. Based on these arguments, we assume that RAC may act as a full mediator between PAC and SPI success. Accordingly, we hypothesize the following:

H3: Realized absorptive capacity fully mediates the relationship between potential absorptive capacity and SPI success.

\subsection{The Moderating Role of Innovation Culture}

According to the literature, IC refers to organizations that are predisposed to learning continuously and that are open to new knowledge or breakthrough solutions to detect and fill gaps between what 
the market desires and what the firm currently offers (Brettel \& Cleven, 2011). When an organization has IC, organizational behaviours facilitate the creation and development of new products, services, or process innovations to address business and environmental challenges and needs (Castro et al., 2013; Ali and Park, 2016). Olmospeñuela et al. (2017) and Aksoy (2017) indicated that IC spurs organizational learning, helps organizations accept new knowledge, technologies, and markets, and determines how they tolerate failure. In addition, IC strengthens organizational cohesion and departmental relationships, which increases the organization's innovation capacity for the adoption of new processes (Nemeth, 1997). An organization's contextual factors also influence its AC mechanisms (Van den Bosch et al., 1999; Harrington and Guimaraes, 2005; Volberda et al., 2010). Specifically, IC enables a firm to increase its openness to external knowledge (Brettel and Cleven, 2011) and helps increase the firm's external learning capacity for knowledge acquisition and assimilation, thereby strengthening high PAC levels (Harrington and Guimaraes, 2005; Winkelbach and Walter, 2015).

In the context of SPI, process improvement may also be viewed as innovation since it encourages a new approach to developing software (Agarwal and Prasad, 2000). During SPI implementation, IC establishes an organizational social environment that facilitates engaging in SPI more creatively, opening the firm to external SPI knowledge. Under a stronger IC, a firm's PAC more aggressively absorbs and filters knowledge to obtain more suitable external SPI knowledge, even when it comes in unfamiliar forms. IC also helps firms better interpret and comprehend the acquired SPI knowledge, which more efficiently and effectively stimulates RAC (Joshi et al., 2010). IC activates an organization's knowledge mechanism to smooth the flow from acquiring external SPI knowledge to utilizing internal SPI knowledge. In this regard, as the level of IC increases, the relationship between PAC and RAC for innovative process improvements strengthens because IC helps the collaboration between PAC and RAC, which better integrates external SPI knowledge into the organization's intelligence. PAC works in conjunction with RAC to improve the efficiency of SPI knowledge transfer, shortening the time to internalize external SPI knowledge and contributing to SPI success. Thus, we suggest that the mediating effect of PAC on SPI success through RAC is moderated by IC and that this effect is stronger for firms with stronger IC. Therefore, we hypothesize the following:

H4: IC positively moderates the relationship between PAC and RAC.

H5: IC moderates the mediating effect of RAC on the relationship between PAC and SPI success.

We propose a theoretical model that integrates PAC, RAC, IC, and SPI success, as shown in Figure 1 , and we conducted an empirical investigation to test the proposed research model and hypotheses.

\section{RESEARCH METHODOLOGY}

\subsection{Data Collection and Sample}

A survey method was adopted to empirically examine the proposed model. Since this study was conducted in Taiwan and China, the questionnaire was prepared and administered in traditional Chinese (for Taiwan) and simplified Chinese (for China), and then, a back-translation procedure was employed to translate the original English versions of the instrument into traditional and simplified Chinese for this paper. To ensure reliability and validity, the traditional and simplified Chinese questionnaires were examined by three IS scholars who are familiar with both the traditional and simplified Chinese language to ensure the survey items were clear, understandable and consistent in meaning. A pilot test was conducted with 10 IS managers from different organizations in China and Taiwan to ensure that the survey questions were understandable.

The organizations that participated in the survey had adopted the CMMI-based SPI program and received official certification from the CMMI institute (see https://sas.cmmiinstitute.com/pars/ pars.aspx). We can observe that organizations that had obtained CMMI certification were either 


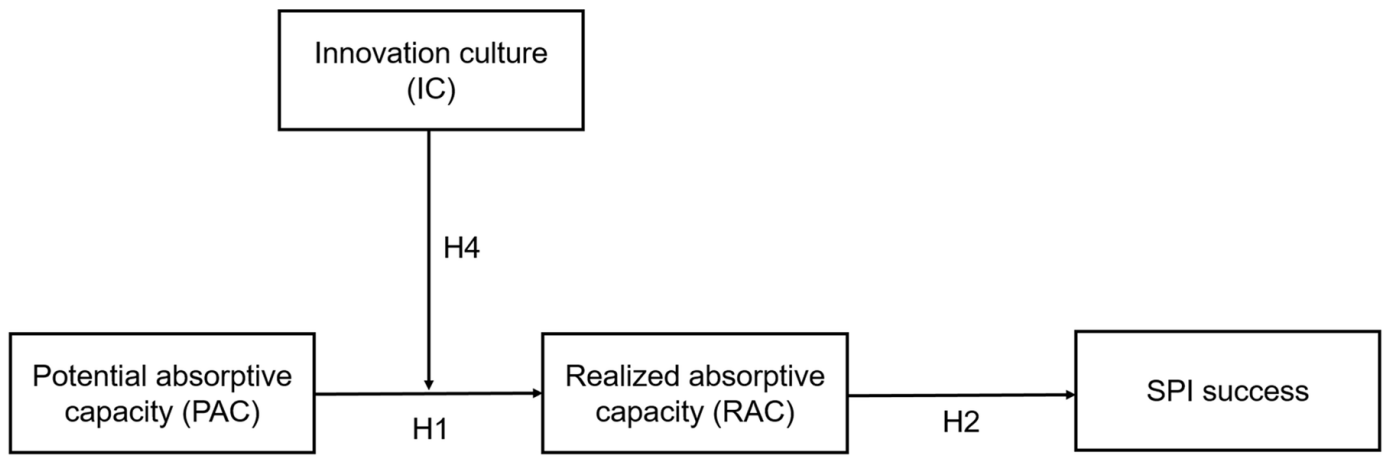

\section{$<$ Descriptions $>$}

H3: RAC fully mediates the relationship between PAC and SPI success.

H5: IC moderates the mediating effect of RAC on the relationship between PAC and SPI success.

software organizations or the software development functions/departments of organizations in different industries. By using the website mentioned above, we first contacted these CMMI-certified organizations by e-mail or telephone and then explained the study's purpose to them to ensure that they understood and agreed to participate. This process was also done to ensure that the participants were serious about answering the questions. As an incentive, the participating organizations were told that they would be provided with our research reports. A total of 200 organizations were willing to participate in the survey. The survey participants were senior and middle management representatives and were directly involved in CMMI implementation at their firms. An electronic mail survey was used for the sample from October to December in 2016, and a total of 200 questionnaires were sent to the CMMI-certified organizations. Since the unit of analysis in this study was at the organizational level, survey participants were asked to answer on behalf of their respective organizations. The respondents returned 125 usable questionnaires (62.5\%). Table 1 in the Appendix shows the demographics of the respondents and organizations.

To check the sample's representativeness, we tested the nonresponse bias using the method of Armstrong and Overton (1977). We conducted t-tests to compare the sample attributes (job position, education, and work experience) of the first $25 \%$ and last $25 \%$ respondents - that is, those who had replied during the first and last seven days. There was no significant difference between these two groups on these items, which indicates that the non-response bias was not significant, and that the representativeness of the samples was supported. Moreover, we performed independent-sample t-tests to compare the means of the same variables for the samples of Chinese firms and Taiwanese firms. The results showed no significant differences between the groups; thus, we can aggregate the data from these two groups.

\subsection{Measures}

Constructs were adapted from those developed and validated in previous studies. All the variables were measured based on a seven-point Likert scale ranging from "strongly disagree" to "strongly agree," as shown in Table 2 in the Appendix. PAC represented a reflective construct including four items that were used to estimate external knowledge acquisition and acquired knowledge assimilation to process newly obtained knowledge. RAC represented a reflective construct comprising four items that were used to estimating knowledge transformation, to combine external knowledge with existing knowledge, and to subsequently apply the new organizational knowledge to achieve a firm's 
objectives. The items for PAC and RAC were adapted from Pavlou and El Sawy (2006). IC was measured using three reflective items adapted from Castro et al. (2013): (1) our firm encourages creativity, innovation, and new ideas in software development processes; (2) our firm encourages experimentation and innovation in improving software development processes; and (3) a common system of values, beliefs, and objectives are related to innovation.

SPI success was measured using a second-order reflective construct formed from two first-order reflective constructs derived from Dyba (2005). The first-order reflective constructs comprised the software organization's perceived SPI success (abbreviated PS) and organizational performance (abbreviated OP). PS was measured as the extent to which SPI was able to (1) substantially increase a firm's software engineering competence and (2) improve a firm's overall performance. The OP construct was measured using three items: (1) the reduction in firm costs, (2) the reduction in the firm's software development cycle time, and (3) the increase in the firm's customer satisfaction. In using these two constructs (i.e., OP and PS), it is worth mentioning that the measurement, particularly the OP in Dyba's (2005) study, was provided with reliability (Cronbach's alpha) to check the internal consistency of the construct, which implies that the study considered cost, cycle time and customer satisfaction to be similar content/indicators that share a common theme: SPI success. This common theme considers that, according to his earlier study (Dyba, 2003), all software processes are expected to help organizations deliver a quality product on schedule and on budget for the ultimate purpose of achieving customer satisfaction. In other words, SPI success is anticipated to help increase OP. In this respect, the construct may serve as a reflective measurement. Based on Jarvis et al. (2003) and Petter et al. (2007), the direction of causality is from construct (i.e., the OP of SPI success in this study) to items (i.e., cost, cycle time and customer satisfaction in this study). Accordingly, we operationalized the OP construct as a reflective construct in this study. For the data analysis in his study, Dyba (2005, p.413) addressed the SPI success construct by averaging the ratings of the two performance dimensions (OP and PS) to form a "single" measure of overall SPI success. Nevertheless, this study used another method to operationalize the second-order construct of SPI success by using two first-order reflective constructs (OP and PS) instead of simply averaging them. The method in this study had a better explanatory power (R-square value) for the model.

Two variables (i.e., firm size and industry types) were controlled in this study. Firm size was regarded as a control variable because large firms may have high-level competencies in software development and can command extra resources (Lee and Chen, 2017, p.7). Therefore, firm size was included in the research model since it may impact organizational SPI success, and it was measured as the natural logarithm of the number of employees. Industry types were controlled because they may produce differences in management and strategies for SPI implementation (Staples et al., 2007).

\section{DATA ANALYSIS AND RESULTS}

The data were empirically examined using PLS structural equation analysis, which is commonly used in the IS literature. PLS is distribution-free, i.e., the estimation is not affected by the complexity of the model, a small sample size, or non-normality in the data; it is orthogonal and overcomes multicollinearity problems (Hair et al., 2013; Lee and Chen, 2017). Analysing a PLS model requires a two-stage approach. The measurement model is first examined to assess reliability and validity, and the structural model is then evaluated to test the relationships among the latent constructs and hypotheses. SmartPLS 3.0 software (Ringle et al, 2015) was employed for the analyses.

\subsection{Measurement Model}

To validate the measurement model, internal consistency, convergent validity, and discriminant validity were assessed. Internal consistency was examined using composite reliability (CR). Table 3 in the Appendix shows that all CR $>0.7$, with a range of 0.789 to 0.885 , which satisfies common 
acceptable levels, as recommended by Fornell and Larcker (1981). Thus, CR was reliable. Convergent validity was assessed by two criteria (Fornell and Larcker, 1981; Hair et al., 2013): all factor loadings should be significant and greater than 0.7 ; and average variance extracted (AVE) for each construct should exceed 0.5 , as it indicates that $50 \%$ or more of the variance was explained by the indicators of the latent variable (Chin, 1998). Table 3 shows that all factor loadings exceed 0.7 and the AVE range was 0.582-0.687, i.e., all AVE were above the recommended level. Thus, the factor loadings and AVEs support convergent validity of the constructs. To confirm discriminant validity, the square root of AVE of a construct should be greater than the correlations between the construct and other constructs in the model (Fornell and Larcker, 1981), as shown in Table 4 in the Appendix. This study also adopted the heterotrait-monotrait ratio of correlations (HTMT) to examine the discriminant validity. All HTMT values were below the accepted value of 0.90 ; hence, discriminant validity has been established between the two variables (Henseler et al., 2015; see Table 4). Tables 3 and 4 show that convergent and discriminant validity are empirically supported, demonstrating the sufficient construct validity of the scales.

The survey data were self-reported, which means common method bias (CMB) may have occurred (Lee and Chen, 2017). To examine CMB, we conducted Harman's one-factor test (Harman, 1967) by performing an exploratory factor analysis (principal components analysis) with all manifest items. All produced factors had eigenvalues greater than 1, and the first factor accounted for only $29.8 \%$ of the total variance. No single factor explained the majority of the variance. Thus, CMB was not significant in this study.

\subsection{Structural Model}

The proposed hypotheses were tested using the bootstrapping technique, and the significance of all hypotheses was examined via 5000 bootstrap runs (Henseler et al., 2016). The test of the structural model included path coefficients and coefficients of determination (R-square). The path coefficients displayed the strength of the associations between the dependent and independent constructs, and the coefficient of determination $\left(\mathrm{R}^{2}\right)$ indicated the amount of variance explained by the independent constructs, representing the predictive power of the model (Lee et al., 2018). Figure 2 shows the main effects, including standardized path coefficients among the constructs, $t$-value and the variance explained. The positive effect of PAC on RAC is significant $(\beta=0.651, \mathrm{p}<0.001)$, supporting H1. Additionally, a positive relationship exists between RAC and SPI success $(\beta=0.336, p<0.001)$, supporting $\mathrm{H} 2$. $\mathrm{R}^{2}$ for SPI success was 0.312 , suggesting $31.2 \%$ of the variance, which exceeded $10 \%$, indicating substantive explanatory power (Bock et al., 2006).

Based on Ajamieh et al.'s (2016) study, we examined the mediating effect by linking the relationship between PAC and SPI success in the research model (see Figure 2). Then, we followed Hair et al. (2013)'s approach to examine H3. We calculated the variance accounted for (VAF), which determined the size of the indirect effect in relation to the total effect, i.e., direct + indirect effects. Therefore, the VAF $=(0.651 * 0.336) /[(0.651 * 0.336)+0.319]=0.407$, i.e., $40.7 \%$ of PAC's effect on SPI success is explained via mediation by RAC. Since $20 \%<\mathrm{VAF}<80 \%$, this effect can be characterized as partial mediation (Hair et al., 2013).

Figure 3 shows that IC positively enhanced the PAC-RAC relationship $(\beta=0.311, \mathrm{p}<0.01)$, supporting H4. Since the proposed model confirmed IC as a moderator of the path from PAC to RAC, the first stage of the mediated effect of PAC on SPI success, a test of moderated mediation is required (Edwards and Lambert, 2007). Accordingly, we conducted Edwards and Lambert's (2007) moderated mediation path analysis. We used Table 5 in the Appendix to illustrate the moderated mediation analysis. Table 5 shows that the indirect effect of PAC on SPI success via RAC was stronger for high IC $(\beta=0.214, \mathrm{p}<0.01)$ than for low IC $(\beta=0.129, \mathrm{p}<0.01)$. Overall, the differences in the indirect effect were significant $(\triangle \beta=0.085, \mathrm{p}<0.05)$, supporting H5. Therefore, IC moderated the mediating effect of RAC on the relationship between PAC and SPI success. 
Figure 2. PLS analysis without moderating effect

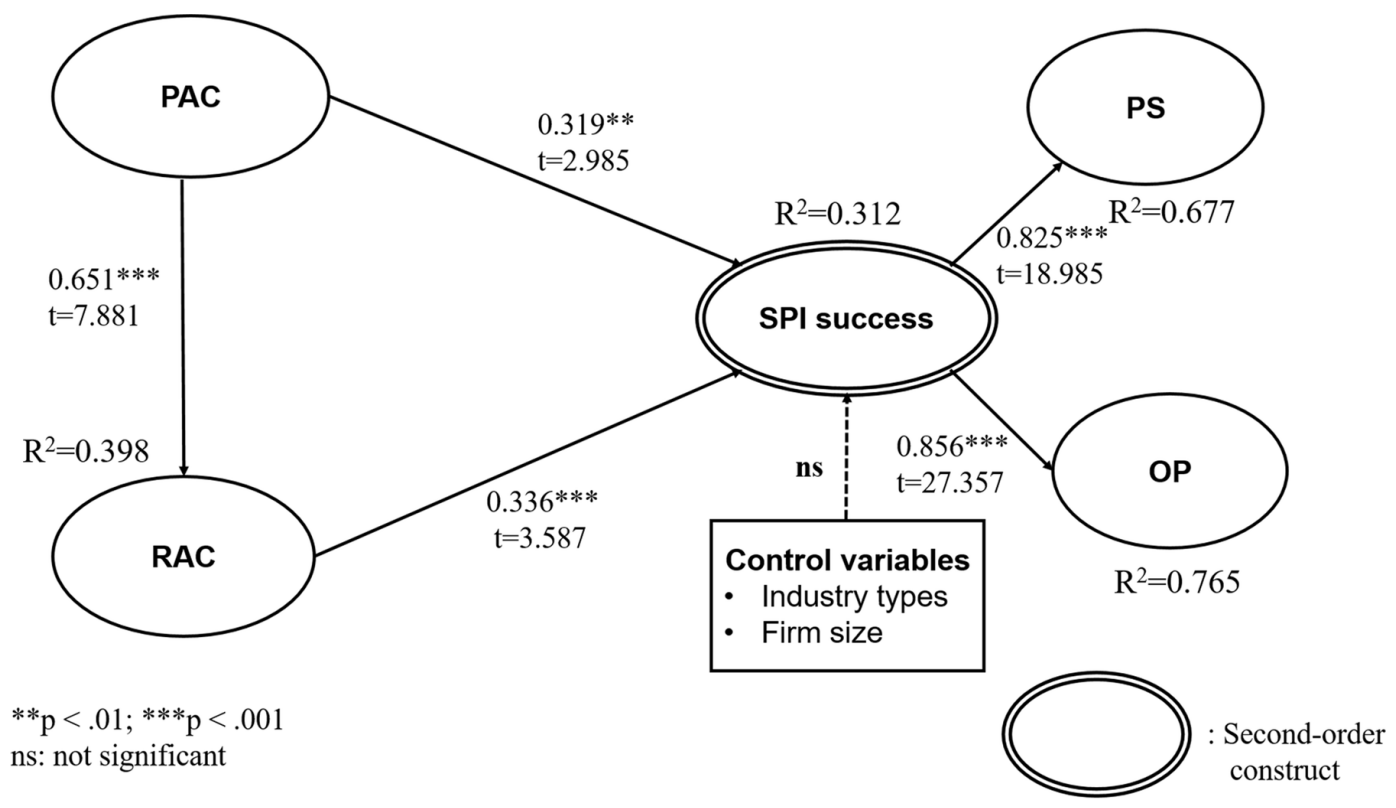

Figure 3. PLS analysis of the moderating effect

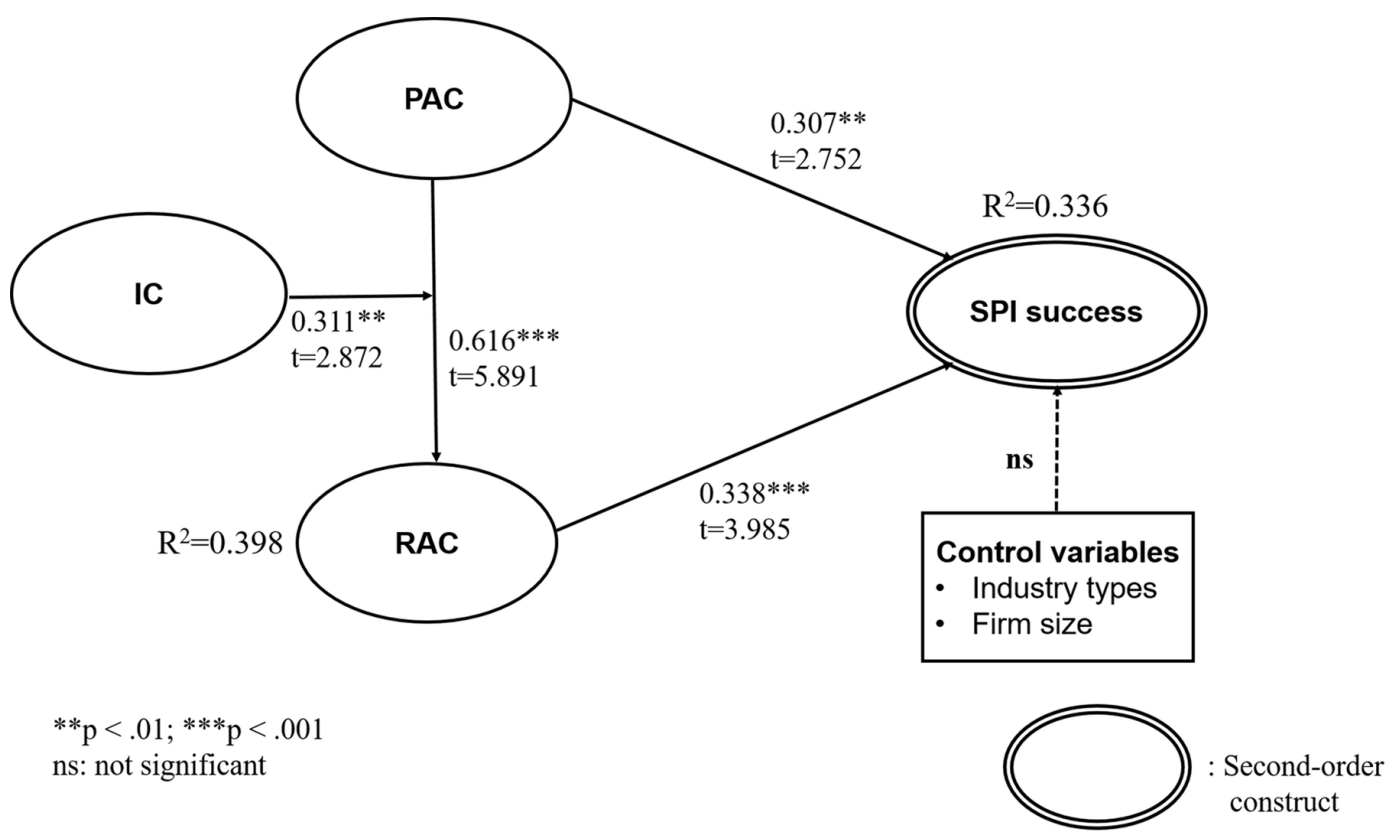

We also examined predictive relevance (Stone-Geisser $\mathrm{Q}^{2}$ ). All the cross-validated redundancy $\mathrm{Q}^{2}$ was greater than 0 (Hair et al., 2013), showing that the constructs of our model possessed predictive relevance. Finally, we used the standardized root mean square residual (SRMR), a measure of the goodness of structural model fit measure for PLS estimations. SRMR $=0.065$, demonstrating that the proposed model was rational (Henseler et al., 2016). 


\section{DISCUSSIONS AND CONCLUSION}

\subsection{Theoretical Contributions}

Our findings offer several important theoretical contributions. First, dynamic capabilities theory and KBV are combined to explain how a firm achieves SPI success through AC and IC. SPI is an organizational learning process (Rus and Lindvall, 2002; Dyba, 2005) and is often aided by external knowledge to support the needs of processes innovation (Ravichandran and Rai, 2003). In the current SPI literature, Dyba (2005) adopted organizational learning theory as a theoretical basis and indicated that a firm's successful SPI implementation depends on the effective exploration of new software development knowledge and the exploitation of existing knowledge. This study extends to further questions and explores how a firm's learning ability internalizes external SPI knowledge. The nature of software development is dynamic, but the organizational learning and related literature does not address this characteristic (e.g., Lee et al., 2016; Mathiassen \& Pourkomeylian, 2003; Niazi et al., 2006; Rus \& Lindvall, 2002; Sulayman et al., 2014), leading to the need for AC to advance our understanding of constant learning and the internalization of externally acquired knowledge.

In determining the influence of dynamic capability on SPI results, Lee et al. (2017) examined $\mathrm{AC}$ in terms of PAC and RAC and studied the mechanisms of SPI success. This study further answers and explains the contextual relationship between PAC and RAC. Strengthening the PACRAC linkage is important for firms in dynamic software development environments because this knowledge-intensive linkage helps firms increase their efficiency when acquiring and internalizing SPI knowledge by addressing changing development needs and the need to expedite this development. Regarding the enhancement of the PAC-RAC relationship, Zahra and George (2002) stated that the use of social integration mechanisms (e.g., offering incentive feedback for the donation and application of ideas or knowledge) can promote the connection between PAC and RAC, increasing the efficiency of AC. Leal-Rodríguez et al. (2014) showed that relational learning plays a moderating role by reinforcing the PAC-RAC link. This study focuses on IC and investigates how it, as a contextual factor, boosts the PAC-RAC association during this attempt. Our empirical results demonstrate that PAC significantly influences RAC, which has a positively significant impact on SPI success and partially mediates the effects of PAC on SPI success. Furthermore, IC positively amplifies the relationship between PAC and RAC.

Second, our empirical results demonstrate that PAC acts as a significant antecedent of RAC. Specifically, PAC enables a firm to prepare for and expose itself to external environments to better gather useful external knowledge; then, RAC is based on PAC to further make use of the absorbed knowledge by addressing and implementing similar SPI needs. Furthermore, RAC operates as a partial intermediary in the relationship between PAC and SPI success, as evidenced by the following empirical situation. In the CMMI-based SPI context, the CMMI reference model provides distinct specific goals (SGs) for process areas (PAs) and recommends corresponding specific practices (SPs) that firms may implement to accomplish the required SGs (SEI, 2010). With PAC, the firm can obtain practical external knowledge (e.g., from consulting firms or exchange conferences) regarding the implementation of SPs. With RAC, the firm can integrate and combine external know-how with the firm's own existing methods to improve and customize practices that meet the firm's goal and accommodate the firm's characteristics. PAC and RAC have different natures and involve distinctive behaviours but are complementary in constructing $\mathrm{AC}$ as a whole, which helps the firm to learn and use external SPI knowledge to accomplish SPI in accordance with a CMMI's goals and practices.

Additionally, the findings of this study do not indicate the expected full mediation between PAC, RAC and SPI success (i.e., H3), which may be because PAC can be seen as a catalyst to induce a firm's intention to engage in behaviour that initiates SPI-related learning activities, a critical premise for a firm aiming to achieve successful SPI implementation. Without PAC, participating firms that conducted SPI by implementing CMMI were unable obtain and select useful external references when learning how to implement the improvement goals and practices specified in the model. This factor 
may also be critical to SPI success. In addition, the empirical evidence implies that PAC possesses the capability to individually foster SPI success. Nevertheless, if firms aim to widely realize and sustain SPI success, RAC is still needed, as it helps the firm to extensively transform and exploit SPI knowledge for similar and future SPI actions.

Finally, compared to a prior study (Lee et al., 2017) that explored and established the relationship between PAC, RAC and SPI success, this study not only further determines the causality of PAC and RAC but also discovers the determinant (IC) to amplify their contribution to SPI success. Specifically, our results show that IC has a critical contextual role to reinforce the path from PAC to RAC. Thus, while the effectiveness of a firm's RAC generally depends on its PAC, this dependence becomes stronger when a higher IC level is present. In software development, which is innovative in nature, process improvements often involve innovative changes or breakthroughs, such as a shift in the development process from a traditional waterfall to an agile approach, which requires the entire organization to buy in. Firms with stronger IC are characterized by intensive communication, embrace of change and a high degree of openness to new or unconventional thoughts or practices (Aksoy, 2017; Olmospeñuela et al., 2017). This situation spurs the firm's propensity toward a dynamic external business environment, helping it become more active in accepting and accommodating external SPI knowledge, which may appear conflicting or inconsistent to the firm. This situation triggers the firm to strengthen the transfer, diffusion and utilization of the newly absorbed SPI knowledge, thus reinforcing RAC mechanisms in terms of transformation and the ability to evolve more effectively. In other words, when a firm has a high level of IC, the synergetic relationship between PAC and RAC is further promoted.

Moreover, stronger IC also amplifies the indirect effect of RAC on the relationship between PAC and SPI success, which implies that IC encourages firms to become actively involved in the quest for external knowledge while simultaneously tightening the link between PAC and RAC, providing greater potency for SPI. This study extends KBV to empirically confirm the conceptual argument of Volberda et al. (2010) that a firm's AC mechanism strengthened under suitable contextual factors, i.e., IC as investigated here, and higher dynamic capability levels, i.e., AC, can be achieved. Thus, a firm with higher AC has relatively efficient SPI knowledge absorption, leading to more positive SPI implementation.

\subsection{Practical Implications}

This study has major managerial implications. First, AC is an interactive process between learning and applying knowledge to improve and enhance organizational capability, and human resources are at the centre of this process (Valentim et al., 2016). A firm's AC resides in employees and can be activated with effective human resource practices. Firms should invest in employees by offering more education and enriching the job design to foster greater AC and should promote advanced competence to keep pace with the dynamic and changing business environment. For example, firms could encourage and sponsor employees to take part in external educational venues, such as conferences, workshops, and professional exhibitions. Effective human resource practices should include job rotation, multi-skill training, and broadly designed jobs in order to broaden employee knowledge in software development and process improvement (Chang et al., 2013) and enable them to absorb knowledge that is not limited to the job scope, which in turn contributes holistically to SPI implementation. In the SPI context, learning from outside the organization requires more than just the acquisition and exploitation of external SPI knowledge. Firms need to update and reconfigure their knowledge stock for superior implementation outcomes. To achieve this, a firm should share its own SPI knowledge with other organizations and thereby receive valuable knowledge feedback. This process will extend a firm's AC to include outside firms' learning abilities.

Second, firms should provide informal means or venues to support the organizational process of implementing acquired SPI knowledge. For example, firms should establish innovative ways to participate more actively in informal knowledge exchanges, e.g., conversations in the cafeteria or 
social media (Facebook, etc.), to stimulate SPI knowledge flows throughout the organization. Social media makes it easier for relevant employees to acquire and understand SPI knowledge. Ooms et al. (2015) showed that social media enables multi-directional and interactive knowledge exchange, which contributes to AC. Thus, encouraging employees to be involved in social media communities helps synthesize acquired SPI knowledge into current organizational knowledge and broadens the utilization of new knowledge when implementing SPI.

Finally, the effect of a firm's PAC on its RAC can be increased by IC since knowing how to apply knowledge to various work domains or different projects requires creativity and imagination. An important role of organizational leadership is to reinforce an active IC, e.g., reward systems must be designed to encourage innovation. Furthermore, assigning activity ownership and giving employees the authority to make decisions regarding trying new things are also encouraged. Firms should embrace divergent thinking, listen to employees, be open to new ideas, and provide employees with appropriate resources in turning new ideas into action. Firms with greater IC will achieve a better response from the dynamic software development environment and consolidate positive SPI implementation outcomes.

\section{LIMITATIONS AND FURTHER RESEARCH}

Despite the above contributions, the current study has several limitations and opportunities for further research. This study included only SPI-certified firms in the Asia-Pacific region; hence, the findings lack generalizability, which is an opportunity for future work. While the CMMI-based SPI-certified samples were appropriate for the research design, this study did not distinguish SPI success in terms of different CMMI maturity levels, i.e., levels 2-5, and the purpose of this study was to investigate the distinct impacts of PAC, RAC and IC on SPI success. Thus, future research should replicate these empirical results for different maturity levels. Finally, because the data in this study are crosssectional in nature, future research should perform a longitudinal study to better establish causality for the hypothesized relationships.

\section{ACKNOWLEDGMENT}

We would like to thank the associate editor and the four reviewers for their constructive comments and suggestions regarding the earlier drafts of this study. The research is supported by the Program for Research Development of Beijing Normal University Zhuhai. 


\section{REFERENCES}

Abubakre, M., Coombs, C. R., \& Ravishankar, M. N. (2017). The impact of salient cultural practices on the outcome of IS implementation. Journal of Global Information Management, 25(1), 1-20. doi:10.4018/ JGIM.2017010101

Agarwal, R., \& Prasad, J. (2000). A field study of the adoption of software process innovations by information systems professionals. IEEE Transactions on Engineering Management, 47(3), 295-308. doi:10.1109/17.865899

Ajamieh, A., Benitez, J., Braojos, J., \& Gelhard, C. (2016). IT infrastructure and competitive aggressiveness in explaining and predicting performance. Journal of Business Research, 69(10), 4667-4674. doi:10.1016/j. jbusres.2016.03.056

Aksoy, H. (2017). How do innovation culture, marketing innovation and product innovation affect the market performance of small and medium-sized enterprises (SMEs)? Technology in Society, 51, 133-141. doi:10.1016/j. techsoc.2017.08.005

Alagarsamy, K., Justus, S., \& Lyakutti, K. (2008). Implementation specification for software process improvement supportive knowledge management tool. IET Software, 2(2), 123-133. doi:10.1049/iet-sen:20070086

Alavi, M., \& Leidner, D. E. (2006). An empirical examination of the influence of organizational culture on knowledge management practices. Journal of Management Information Systems, 22(3), 191-224. doi:10.2753/ MIS0742-1222220307

Albort-Morant, G., Leal-Rodríguez, A. L., Henseler, J., \& Cepeda-Carrion, G. A. (2018). Potential and realized absorptive capacity as complementary drivers of green product and process innovation performance. Sustainability, 10(2), 381. doi:10.3390/su10020381

Ali, M., \& Park, K. (2016). The mediating role of an innovative culture in the relationship between absorptive capacity and technical and non-technical innovation. Journal of Business Research, 43(4), 1680-1687.

Armstrong, J. S., \& Overton, T. S. (1977). Estimating nonresponse bias in mail surveys. JMR, Journal of Marketing Research, 14(3), 396-402. doi:10.2307/3150783

Bharati, P., Zhang, C., \& Chaudhury, A. (2014). Social media assimilation in firms: Investigating the roles of absorptive capacity and institutional pressures. Information Systems Frontiers, 16(2), 257-27. doi:10.1007/ s10796-013-9433-x

Bock, G. W., Kankanhalli, A., \& Sharma, S. (2006). Are norms enough? the role of collaborative norms in promoting organizational knowledge seeking. European Journal of Information Systems, 15(4), 357-367. doi:10.1057/palgrave.ejis.3000630

Brettel, M., \& Cleven, N. J. (2011). Innovation culture, collaboration with external partners and NPD performance. Creativity and Innovation Management, 20(4), 253-272. doi:10.1111/j.1467-8691.2011.00617.x

Camisón, C., \& Forés, B. (2010). Knowledge absorptive capacity: New insights for its conceptualization and measurement. Journal of Business Research, 63(7), 707-715. doi:10.1016/j.jbusres.2009.04.022

Castro, M. D., Delgado-Verde, M., Navas-López, J. E., \& Cruz-González, J. (2013). The moderating role of innovation culture in the relationship between knowledge assets and product innovation. Technological Forecasting and Social Change, 80(2), 351-363. doi:10.1016/j.techfore.2012.08.012

Chang, S., Gong, Y., Way, S. A., \& Jia, L. (2013). Flexibility-oriented FRM systems, absorptive capacity, and market responsiveness and firm innovativeness. Journal of Management, 39(7), 1924-1951. doi: $10.1177 / 0149206312466145$

Joshi, K. D., Chi, L., Datta, A., \& Han, S. (2010). Changing the competitive landscape: Continuous innovation through it-enabled knowledge capabilities. Information Systems Research, 21(3), 472-495. doi:10.1287/ isre. 1100.0298

CMMI Institute. (2010). CMMI for development quick reference. Retrieved from https://cmmiinstitute.com/ getattachment/6807c668-a92d-405a-894d-014876dcafb9/attachment.aspx 
Cohen, W. M., \& Levinthal, D. A. (1990). Absorptive capacity: A new perspective on learning and innovation. Administrative Science Quarterly, 35(1), 128-152. doi:10.2307/2393553

Dyba, T. (2003). A dynamic model of software engineering knowledge creation. Managing software engineering knowledge. Springer Berlin Heidelberg. 95-117.

Dyba, T. (2005). An empirical investigation of the key factors for success in software process improvement. IEEE Transactions on Software Engineering, 31(5), 410-424. doi:10.1109/TSE.2005.53

Edwards, J. R., \& Lambert, L. S. (2007). Methods for integrating moderation and mediation: A general analytical framework using moderated path analysis. Psychological Methods, 12(1), 1-22. doi:10.1037/1082-989X.12.1.1 PMID:17402809

Egorova, E., Torchiano, M., \& Morisio, M. (2009). Evaluating the perceived effect of software engineering practices in the Italian industry. In Trustworthy Software Development Processes (pp. 100-111). Springer Berlin Heidelberg. doi:10.1007/978-3-642-01680-6_11

Feher, P., \& Gabor, A. (2010). The role of knowledge management supporters in software development companies. Software Process Improvement and Practice, 11(3), 251-260. doi:10.1002/spip.269

Fornell, C., \& Larcker, D. F. (1981). Evaluating structural equation models with unobservable variables and measurement error. JMR, Journal of Marketing Research, 18(1), 39-50. doi:10.2307/3151312

Fosfuri, A., \& Tribó, J. A. (2008). Exploring the antecedents of potential absorptive capacity and its impact on innovation performance. Omega, 36(2), 173-187. doi:10.1016/j.omega.2006.06.012 PMID:18680889

Grant, R. M. (1996). Toward a knowledge-based theory of the firm. Strategic Management Journal, 17(S2), 109-122. doi:10.1002/smj.4250171110

Hair, J. F., Hult, G. T. M., Ringle, C., \& Sarstedt, M. (2013). A primer on partial least squares structural equation modeling (PLS-SEM). SAGE Publications.

Harman, H. (1967). Modern factor analysis. Chicago: University of Chicago Press.

Harrington, S. J., \& Guimaraes, T. (2005). Corporate culture, absorptive capacity and it success. Information and Organization, 15(1), 39-63. doi:10.1016/j.infoandorg.2004.10.002

Henseler, J., Hubona, G., \& Ray, P. A. (2016). Using PLS path modeling in new technology research: Updated guidelines. Industrial Management \& Data Systems, 116(1), 2-20. doi:10.1108/IMDS-09-2015-0382

Henseler, J., Ringle, C. M., \& Sarstedt, M. (2015). A new criterion for assessing discriminant validity in variance-based structural equation modeling. Journal of the Academy of Marketing Science, 43(1), 115-135. doi:10.1007/s11747-014-0403-8

Jansen, J. J. P., Bosch, F. A. J., \& Volberda, H. W. (2005). Managing potential and realized absorptive capacity: How do organizational antecedents matter? Academy of Management Journal, 48(6), 999-1015. doi:10.5465/ amj.2005.19573106

Janz, B. D., \& Prasarnphanich, P. (2003). Understanding the antecedents of effective knowledge management: The importance of a knowledge-centered culture. Decision Sciences, 34(2), 351-384. doi:10.1111/1540-5915.02328

Jarvis, C. B., Mackenzie, S. B., \& Podsakoff, P. M. (2003). A critical review of construct indicators and measurement model misspecification in marketing and consumer research. The Journal of Consumer Research, 30(2), 199-218. doi:10.1086/376806

Jones, M. C., Cline, M., \& Ryan, S. (2006). Exploring knowledge sharing in ERP implementation: An organizational culture framework. Decision Support Systems, 41(2), 411-434. doi:10.1016/j.dss.2004.06.017

Ke, W., \& Wei, K. K. (2008). Organizational culture and leadership in ERP implementation. Decision Support Systems, 45(2), 208-218. doi:10.1016/j.dss.2007.02.002

Ko, D. G., Kirsch, L. J., \& King, W. R. (2005). Antecedents of knowledge transfer from consultants to clients in enterprise system implementations. Management Information Systems Quarterly, 29(1), 59-85. doi: $10.2307 / 25148668$ 
Lane, P. J., Koka, B. R., \& Pathak, S. (2006). The reification of absorptive capacity: A critical review and rejuvenation of the construct. Academy of Management Review, 31(4), 833-863. doi:10.5465/amr.2006.22527456

Larrucea, X., O'Connor, R. V., Colomopalacios, R., \& Laporte, C. Y. (2016). Software process improvement in very small organizations. IEEE Software, 33(2), 85-89. doi:10.1109/MS.2016.42

Leal-Rodríguez, A. L., Roldán, J. L., Ariza-Montes, J. A., \& Leal-Millán, A. (2014). From potential absorptive capacity to innovation outcomes in project teams: The conditional mediating role of the realized absorptive capacity in a relational learning context. International Journal of Project Management, 32(6), 894-907. doi:10.1016/j.ijproman.2014.01.005

Lee, J. C., \& Chen, C. Y. (2017). Exploring the determinants of software process improvement success: A dynamic capability view. Information Development, 2017. doi:10.1177/0266666917724194

Lee, J. C., Chen, C. Y., \& Shiue, Y. C. (2017). The moderating effects of organisational culture on the relationship between absorptive capacity and software process improvement success. Information Technology \& People, 30(1), 47-70. doi:10.1108/ITP-09-2013-0171

Lee, J. C., Hsu, W. C., \& Chen, C. Y. (2018). Impact of absorptive capability on software process improvement and firm performance. Information Technology Management, 19(1), 21-35. doi:10.1007/s10799-016-0272-6

Lee, J. C., Shiue, Y. C., \& Chen, C. Y. (2016). Examining the impacts of organizational culture and top management support of knowledge sharing on the success of software process improvement. Computers in Human Behavior, 54, 462-474. doi:10.1016/j.chb.2015.08.030

Lee, W. T., Hung, S.-Y., \& Chau, P. Y. K. (2011). Influence of knowledge management infrastructure on innovative business processes and market-interrelationship performance: An empirical study of hospitals in Taiwan. Journal of Global Information Management, 19(2), 67-89. doi:10.4018/jgim.2011040104

Leidner, D. E., \& Kayworth, T. (2006). Review: a review of culture in information systems research: toward a theory of information technology culture conflict. Management Information Systems Quarterly, 30(30), 357-399. doi: $10.2307 / 25148735$

March, J. G. (1991). Exploration and exploitation in organizational learning. Organization Science, 2(1), 71-87. doi:10.1287/orsc.2.1.71

Mathiassen, L., \& Pourkomeylian, P. (2003). Managing knowledge in a software organization. Journal of Knowledge Management, 7(2), 63-80. doi:10.1108/13673270310477298

Matusik, S. F., \& Heeley, M. B. (2005). Absorptive capacity in the software industry: Identifying dimensions that affect knowledge and knowledge creation activities. Journal of Management, 31(4), 549-572. doi: $10.1177 / 0149206304272293$

Meehan, B., \& Richardson, I. (2010). Identification of software process knowledge management. Software Process Improvement and Practice, 7(2), 47-55. doi:10.1002/spip.154

Moreno, V., Pinheiro, J. R. M., \& Joia, L. A. (2012). Resource-based view, knowledge-based view and the performance of software development companies: A study of Brazilian SMEs. Journal of Global Information Management, 20(4), 27-53. doi:10.4018/jgim.2012100102

Mueller, J. (2014). A specific knowledge culture: Cultural antecedents for knowledge sharing between project teams. European Management Journal, 32(2), 190-202. doi:10.1016/j.emj.2013.05.006

Nemeth, C. J. (1997). Managing innovation: When less is more. California Management Review, 40(1), 59-74. doi: $10.2307 / 41165922$

Niazi, M., Wilson, D., \& Zowghi, D. (2010). Critical success factors for software process improvement implementation: An empirical study. Software Process Improvement and Practice, 11(2), 193-211. doi:10.1002/ spip.261

Nonaka, I., \& Takeuchi, H. (1995). The knowledge-creating company: how Japanese companies create the dynamics of innovation. New York: Oxford University Press. 
Olmospeñuela, J., Garcíagranero, A., Castromartínez, E., \& D’Este, P. (2017). Strengthening smes’ innovation culture through collaborations with public research organizations. do all firms benefit equally? European Planning Studies. doi:10.1080/09654313.2017.1279592

Ooms, W., Bell, J., \& Kok, R. A. W. (2015). Use of social media in inbound open innovation: Building capabilities for absorptive capacity. Creativity and Innovation Management, 24(1), 136-150. doi:10.1111/caim.12105

Pavlou, P. A., \& Sawy, O. A. E. (2006). From it leveraging competence to competitive advantage in turbulent environments: The case of new product development. Information Systems Research, 17(3), 198-227. doi:10.1287/ isre. 1060.0094

Peng, G., Dey, D., \& Lahiri, A. (2014). Healthcare it adoption: An analysis of knowledge transfer in socioeconomic networks. Journal of Management Information Systems, 31(3), 7-34. doi:10.1080/07421222.2014.994672

Petter, S., Straub, D., \& Rai, A. (2007). Specifying formative constructs in information systems research. Management Information Systems Quarterly, 31(4), 623-656. doi:10.2307/25148814

Rainer, A., \& Hall, T. (2002). Key success factors for implementing software process improvement: A maturitybased analysis. Journal of Systems and Software, 62(2), 71-84. doi:10.1016/S0164-1212(01)00122-4

Ravichandran, T., \& Rai, A. (2003). Structural analysis of the impact of knowledge creation and knowledge embedding on software process capability. IEEE Transactions on Engineering Management, 50(3), $270-284$. doi:10.1109/TEM.2003.817278

Ringle, C. M., Wende, S., \& Becker, J. M. (2015). SmartPLS 3. Boenningstedt: SmartPLS GmbH. Retrieved from http://www.smartpls.com

Roberts, N. H., Galluch, P. S., Dinger, M., \& Grover, V. (2012). Absorptive capacity and information systems research: Review, synthesis, and directions for future research. Management Information Systems Quarterly, $36(2), 625-648$.

Rus, I., \& Lindvall, M. (2002). Guest editors' introduction: Knowledge management in software engineering. IEEE Software, 19(3), 26-38. doi:10.1109/MS.2002.1003450

Saemundsson, R. J., \& Candi, M. (2017). Absorptive capacity and the identification of opportunities in new technology-based firms. Technovation, 64, 43-49. doi:10.1016/j.technovation.2017.06.001

Saraf, N., Liang, H., Xue, Y., \& Hu, Q. (2013). How does organisational absorptive capacity matter in the assimilation of enterprise information systems? Information Systems Journal, 23(3), 245-267. doi:10.1111/j.13652575.2011.00397.x

Sharma, S., Daniel, E. M., \& Gray, C. (2012). Absorptive capacity and ERP implementation in Indian mediumsized firms. Journal of Global Information Management, 20(4), 54-79. doi:10.4018/jgim.2012100103

Shih, C. C., \& Huang, S. J. (2010). Exploring the relationship between organizational culture and software process improvement deployment. Information \& Management, 47(5), 271-281. doi:10.1016/j.im.2010.06.001

Staples, M., Niazi, M., Jeffery, R., Abrahams, A., Byatt, P., \& Murphy, R. (2007). An exploratory study of why organizations do not adopt CMMI. Journal of Systems and Software, 80(6), 883-895. doi:10.1016/j. jss.2006.09.008

Sulayman, M., Mendes, E., Urquhart, C., Riaz, M., \& Tempero, E. (2014). Towards a theoretical framework of SPI success factors for small and medium web companies. Information and Software Technology, 56(7), 807-820. doi:10.1016/j.infsof.2014.02.006

Sun, P. Y. T., \& Anderson, M. H. (2010). An examination of the relationship between absorptive capacity and organizational learning, and a proposed integration. International Journal of Management Reviews, 12(2), 130-150. doi:10.1111/j.1468-2370.2008.00256.x

Teece, D. J., Pisano, G. P., \& Shuen, A. (1997). Dynamic capabilities and strategic management. Strategic Management Journal, 18(7), 509-533. doi:10.1002/(SICI)1097-0266(199708)18:7<509::AIDSMJ882>3.0.CO;2-Z 
Uskarci, A., \& Demirors, O. (2017). Do staged maturity models result in organization-wide continuous process improvement? insight from employees. Computer Standards \& Interfaces, 52, 25-40. doi:10.1016/j. csi.2017.01.008

Valentim, L., Lisboa, J. V., \& Franco, M. (2016). Knowledge management practices and absorptive capacity in small and medium-sized enterprises: Is there really a linkage? $R \& D$ Management, 46(4), 711-725. doi:10.1111/ $\operatorname{radm} .12108$

Volberda, H. W., Foss, N. J., \& Lyles, M. A. (2010). Absorbing the concept of absorptive capacity: How to realize its potential in the organization field. Organization Science, 21(4), 931-951. doi:10.1287/orsc.1090.0503

Wei, J., Lowry, P. B., \& Seedorf, S. (2015). The assimilation of RFID technology by Chinese companies: A technology diffusion perspective. Information \& Management, 52(6), 628-642. doi:10.1016/j.im.2015.05.001

Winkelbach, A., \& Walter, A. (2015). Complex technological knowledge and value creation in science-to-industry technology transfer projects: The moderating effect of absorptive capacity. Industrial Marketing Management, 47, 98-108. doi:10.1016/j.indmarman.2015.02.035

Winter, J., \& Rönkkö, K. (2010). SPI success factors within product usability evaluation. Journal of Systems and Software, 83(11), 2059-2072. doi:10.1016/j.jss.2010.04.066

Xu, P., \& Ramesh, B. (2007). Software process tailoring: An empirical investigation. Journal of Management Information Systems, 24(2), 293-328. doi:10.2753/MIS0742-1222240211

Zahra, S. A., \& George, G. (2002). Absorptive capacity: A review, reconceptualization, and extension. Academy of Management Review, 27(2), 185-203. doi:10.5465/amr.2002.6587995

Zahra, S. A., Sapienza, H. J., \& Davidsson, P. (2006). Entrepreneurship and dynamic capabilities: A review, model and research agenda. Journal of Management Studies, 43(4), 917-955. doi:10.1111/j.1467-6486.2006.00616.x 


\section{APPENDIX A}

Table 1. Characteristics of the samples ( $\mathrm{N}=68$ from Taiwan, $\mathrm{N}=57$ from Mainland China)

\begin{tabular}{|c|c|c|}
\hline \multicolumn{3}{|c|}{ Characteristics of the Organizations } \\
\hline Industry type & Frequency & Percentage \\
\hline Information technology & 70 & $56.0 \%$ \\
\hline Manufacturing & 36 & $28.8 \%$ \\
\hline Research institute & 8 & $6.4 \%$ \\
\hline Finance & 6 & $4.8 \%$ \\
\hline Education & 3 & $2.4 \%$ \\
\hline Health care & 2 & $1.6 \%$ \\
\hline \multicolumn{3}{|l|}{ Number of employees } \\
\hline Below 50 & 30 & $24.0 \%$ \\
\hline $50-100$ & 36 & $28.8 \%$ \\
\hline $100-500$ & 32 & $25.6 \%$ \\
\hline $500-1000$ & 17 & $13.6 \%$ \\
\hline Above 1000 & 10 & $8.0 \%$ \\
\hline \multicolumn{3}{|c|}{ Characteristics of the Respondents } \\
\hline \multicolumn{3}{|l|}{ Job Position } \\
\hline CEO & 5 & $4.0 \%$ \\
\hline Vice/Assistant president & 9 & $7.2 \%$ \\
\hline General manager & 36 & $28.8 \%$ \\
\hline Manager & 75 & $60.0 \%$ \\
\hline \multicolumn{3}{|l|}{ Education } \\
\hline Bachelor's degree & 42 & $33.6 \%$ \\
\hline Master's degree & 64 & $51.2 \%$ \\
\hline Doctorate & 19 & $15.2 \%$ \\
\hline \multicolumn{3}{|l|}{ Work Experience } \\
\hline $1-5$ years & 16 & $12.8 \%$ \\
\hline $6-10$ years & 61 & $48.8 \%$ \\
\hline $11-15$ years & 31 & $24.8 \%$ \\
\hline $16-20$ years & 10 & $8.0 \%$ \\
\hline Above 21 years & 7 & $5.6 \%$ \\
\hline
\end{tabular}


Table 2. Measurement instrument

\begin{tabular}{|c|c|c|}
\hline Construct & Items & $\begin{array}{l}\text { Adapted } \\
\text { From }\end{array}$ \\
\hline \multirow{4}{*}{$\begin{array}{l}\text { Potential } \\
\text { absorptive } \\
\text { capacity (PAC) }\end{array}$} & (PAC1) We are able to identify and acquire internal and external knowledge. & \multirow{8}{*}{$\begin{array}{l}\text { Pavlou and } \\
\text { El Sawy } \\
(2006)\end{array}$} \\
\hline & (PAC2) We have routines to identify, value, and import new information and knowledge. & \\
\hline & (PAC3) We have adequate routines to analyse the information and knowledge obtained. & \\
\hline & (PAC4) We have adequate routines to assimilate new information and knowledge. & \\
\hline \multirow{4}{*}{$\begin{array}{l}\text { Realized } \\
\text { absorptive } \\
\text { capacity (RAC) }\end{array}$} & (RAC1) We can successfully integrate our existing information into new knowledge. & \\
\hline & (RAC2) We are effective in transforming existing information into new knowledge. & \\
\hline & (RAC3) We can successfully exploit internal and external information and knowledge into concrete applications. & \\
\hline & (RAC4) We are effective in utilizing knowledge into new products or services. & \\
\hline \multirow{2}{*}{$\begin{array}{l}\text { Perceived level of } \\
\text { SPI success (PS) }\end{array}$} & (PS1) Our SPI work has substantially increased our software engineering competence. & \multirow{5}{*}{$\begin{array}{l}\text { Dyba } \\
(2005)\end{array}$} \\
\hline & (PS2) Our SPI work has substantially improved our overall performance. & \\
\hline \multirow{3}{*}{$\begin{array}{l}\text { Organizational } \\
\text { performance }(\mathrm{OP})\end{array}$} & (OP1) Over the past three years, we have greatly reduced the cost of software development. & \\
\hline & (OP2) Over the past three years, we have greatly reduced the cycle time of software development. & \\
\hline & (OP3) Over the past three years, we have greatly increased our customers' satisfaction. & \\
\hline \multirow{3}{*}{$\begin{array}{l}\text { Innovation culture } \\
\text { (IC) }\end{array}$} & $\begin{array}{l}\text { (IC1) Our company encourages creativity, innovation and/or the development of new ideas in software } \\
\text { development processes. }\end{array}$ & \multirow{3}{*}{$\begin{array}{l}\text { Castro et al. } \\
(2013)\end{array}$} \\
\hline & (IC2) A common system of values, beliefs and objectives exists in our company, directed towards innovation. & \\
\hline & (IC3) Our company encourages experimentation and innovation in order to improve software development processes. & \\
\hline
\end{tabular}

Table 3. Measurement model results

\begin{tabular}{|c|c|c|c|}
\hline Construct/Indicator & Factor Loadings & AVE & CR \\
\hline Potential absorptive capacity (PAC) & & 0.582 & 0.847 \\
\hline $\mathrm{PAC} 1$ & 0.712 & & \\
\hline PAC2 & 0.731 & & \\
\hline PAC3 & 0.788 & & \\
\hline PAC4 & 0.815 & & \\
\hline Realized absorptive capacity (RAC) & & 0.658 & 0.885 \\
\hline $\mathrm{RAC1}$ & 0.786 & & \\
\hline $\mathrm{RAC} 2$ & 0.830 & & \\
\hline RAC3 & 0.812 & & \\
\hline RAC4 & 0.815 & & \\
\hline Level of perceived SPI success (PS) & & 0.651 & 0.789 \\
\hline PS1 & 0.836 & & \\
\hline PS2 & 0.777 & & \\
\hline Organizational performance (OP) & & 0.584 & 0.808 \\
\hline OP1 & 0.719 & & \\
\hline $\mathrm{OP} 2$ & 0.758 & & \\
\hline OP3 & 0.812 & & \\
\hline Innovation culture (IC) & & 0.687 & 0.868 \\
\hline $\mathrm{IC} 1$ & 0.785 & & \\
\hline IC2 & 0.876 & & \\
\hline IC3 & 0.823 & & \\
\hline
\end{tabular}


Table 4. Correlation of the constructs, the square root of AVEs and the HTMT

\begin{tabular}{|l|l|l|l|l|l|}
\hline \multicolumn{1}{|c|}{ Construct } & \multicolumn{1}{c|}{ OP } & \multicolumn{1}{c|}{ PAC } & \multicolumn{1}{c|}{ PS } & \multicolumn{1}{c|}{ RAC } & IC \\
\hline OP & $\mathbf{0 . 7 6 4}$ & & & & \\
\hline PAC & $0.468(0.522)$ & $\mathbf{0 . 7 6 3}$ & & & \\
\hline PS & $0.412(0.758)$ & $0.372(0.228)$ & $\mathbf{0 . 8 0 7}$ & & \\
\hline RAC & $0.489(0.558)$ & $0.539(0.605)$ & $0.351(0.209)$ & $\mathbf{0 . 8 1 1}$ & \\
\hline IC & $0.377(0.309)$ & $0.455(0.511)$ & $0.568(0.612)$ & $0.365(0.217)$ & $\mathbf{0 . 8 2 9}$ \\
\hline
\end{tabular}

Note: Square root of AVEs on diagonal in boldface. The values in parentheses are the HTMT.

Table 5. Moderated mediation path analysis

\begin{tabular}{|l|l|l|l|l|l|}
\hline \multirow{2}{*}{ Moderating Variable } & \multicolumn{5}{|c|}{ PAC $(\mathbf{X}) \rightarrow$ RAC $(\mathbf{M}) \rightarrow$ SPI Success $(\mathbf{Y})$} \\
\cline { 2 - 6 } & \multicolumn{2}{|c|}{ Stage } & \multicolumn{3}{c|}{ Effect } \\
\cline { 2 - 6 } & \multicolumn{1}{|c|}{$\begin{array}{c}\text { First } \\
\left(\mathbf{P}_{M X}\right)\end{array}$} & $\begin{array}{c}\text { Second } \\
\left(\mathbf{P}_{Y M}\right)\end{array}$ & $\begin{array}{c}\text { Direct } \\
\left(\mathbf{P}_{Y X}\right)\end{array}$ & $\begin{array}{c}\text { Indirect } \\
\left(\mathbf{P}_{Y M} \mathbf{P}_{M X}\right)\end{array}$ & $\begin{array}{c}\text { Total } \\
\left(\mathbf{P}_{Y X}+\mathbf{P}_{Y M} \mathbf{P}_{M X}\right)\end{array}$ \\
\hline High levels of innovation culture & $0.625^{* * *}$ & $0.342^{* * *}$ & $0.317^{* *}$ & $0.214^{* *}$ & $0.531^{* *}$ \\
\hline Low levels of innovation culture & $0.392^{* *}$ & $0.330^{* * *}$ & $0.302^{* *}$ & $0.129^{* *}$ & $0.431^{* *}$ \\
\hline Differences & $0.033^{*}$ & $0.12^{*}$ & 0.015 & $0.085^{*}$ & $0.10^{*}$ \\
\hline
\end{tabular}

Note: $\mathrm{N}=125 ;{ }^{*} \mathrm{p}<0.05 ;{ }^{* *} \mathrm{p}<0.01$; ${ }^{* * *} \mathrm{p}<0.001$ (two-tailed test); PAC = potential absorptive capacity; $\mathrm{RAC}=$ realized absorptive capacity; $S P I=$ software process improvement. $P_{M X}=$ path from PAC to RAC; $P_{Y M}=$ path from RAC to SPI success; $P_{Y X}=$ path from PAC to SPI success; Tests of differences for the indirect and total effect were based on bias-corrected confidence intervals derived from bootstrap estimates.

Jung-Chieh Lee is an assistant professor in the International Business Faculty at Beijing Normal University Zhuhai in China. His research interests include knowledge management, organizational behavior, and software process improvement. His papers have been published or accepted in Computers in Human Behavior, Information Technology and People, Information Technology and Management and Information Development.

Chung-Yang Chen is a distinguished professor at the Department of Information Management, National Central University, Taiwan. His research interests include the enhancement of information systems, such as software process improvement, project management and quality management systems, such as data/information quality, software quality and software engineering education. His research has been published in many journals such as Journal of System and Software, Information Technology \& People, European Journal of Operational Research, Computers in Human Behavior, Journal of Engineering and Technology Management, International Journal of Project Management, IIE Transactions, Total Quality Management and Business Excellence, IEEE Transactions on Education, Evaluation \& Assessment in Higher Education, etc. 